\title{
APPROXIMATE MAPS CHARACTERIZING INJECTIVITY AND SURJECTIVITY OF MAPS
}

\author{
TAKAHISA MiYATA
}

Kobe University, Japan

\begin{abstract}
In the theory of inverse systems, in order to study the properties of a space $X$ or a map $f: X \rightarrow Y$ between spaces, one expands $X$ to an inverse system $\boldsymbol{X}$ or expands $f$ to a map $\boldsymbol{f}: \boldsymbol{X} \rightarrow \boldsymbol{Y}$ between the inverse systems, and then work on $\boldsymbol{X}$ or $\boldsymbol{f}$. In this paper, we define approximate injectivity (resp., surjectivity) for approximate maps, and show that a map $f: X \rightarrow Y$ between compact metric spaces is injective (resp., surjective) if and only if any approximate map $f: \mathfrak{X} \rightarrow \mathfrak{Y}$ whose limit is $f$ is injective (resp., surjective). As a consequence, we show that an approximate map $\boldsymbol{f}: \mathfrak{X} \rightarrow \mathfrak{Y}$ is approximately injective (resp., approximately surjective) if and only if $\boldsymbol{f}$ represents a monomorphism (resp., an epimorphism) in the approximate pro-category in the sense of Mardešić and Watanabe.
\end{abstract}

\section{INTRODUCTION}

In the theory of inverse systems, given a space $X$ or a map $f: X \rightarrow Y$, one uses an inverse system or a map between inverse systems to get information on the space $X$ or the map $f$. More precisely, given a space $X$, one of the typical ways is to expand $X$ into a resolution $\boldsymbol{p}: X \rightarrow \boldsymbol{X}$ in the sense of S. Mardešić ([4]) (or an approximate resolution $\boldsymbol{p}: X \rightarrow \mathfrak{X}$ in the sense of Mardešić and T. Watanabe ([8])) and study the inverse system $\boldsymbol{X}$ (or the approximate inverse system $\mathfrak{X}$ ) to obtain the properties of $X$. In a similar way, given a map $f: X \rightarrow Y$, one considers a map $\boldsymbol{f}: \boldsymbol{X} \rightarrow \boldsymbol{Y}$ between the inverse systems (or an approximate map $f: \mathfrak{X} \rightarrow \mathfrak{Y}$ between the approximate inverse systems) and study $f$ to obtain the properties of $f$.

2010 Mathematics Subject Classification. 54C56, 54C25, 54B30.

Key words and phrases. Injective map, surjective map, epimorphism, monomorphism, approximate map, shape. 
For example, every map $f: X \rightarrow Y$ between compact metric spaces admits compact polyhedral inverse sequences $\boldsymbol{X}=\left(X_{i}, p_{i, i+1}\right)$ and $\boldsymbol{Y}=$ $\left(Y_{j}, q_{j, j+1}\right)$ and maps of inverse sequences $\boldsymbol{f}=\left(f_{j}, \varphi\right): \boldsymbol{X} \rightarrow \boldsymbol{Y}$ whose limit is $f$, where $\varphi: \mathbb{N} \rightarrow \mathbb{N}$ is an increasing function. Here $\boldsymbol{f}$ is a map if for $j<j^{\prime}$, there exists $i>\varphi\left(j^{\prime}\right)$ such that

$$
f_{j} p_{\varphi(j) i}=q_{j j^{\prime}} f_{j^{\prime}} p_{\varphi\left(j^{\prime}\right) i},
$$

and $f$ is the limit of $\boldsymbol{f}$ if the following equality holds:

$$
f_{j} p_{\varphi(j)}=q_{j} f, \text { for } j \in \mathbb{N} .
$$

However, if the polyhedral inverse sequences $\boldsymbol{X}$ and $\boldsymbol{Y}$ are chosen in advance, there may not exist maps $f_{j}: X_{\varphi(j)} \rightarrow Y_{j}$ satisfying both (1.1) and (1.2). In order to overcome this deficiency, Watanabe ([9]) introduced the notion of approximate map (approximative map in the literature). An approximate map differs from the usual map of inverse sequences in the sense that it requires only approximate commutativity in stead of the commutativity relation (1.1).

In this paper, we introduce the notion of approximately injectivity (resp., approximately surjectivity) for approximate maps. The purpose of this paper is to show that a map $f: X \rightarrow Y$ between compact metric spaces is injective (resp., surjective) if and only if for any approximate map $f: \mathfrak{X} \rightarrow \mathfrak{Y}$ whose limit is $f, \boldsymbol{f}$ is approximately injective (resp., surjective) (Theorem 3.1 (resp., Theorem 4.1)). The part for surjectivity was proved for approximate maps between noncommutative approximate inverse sequences in [3], here we give a simpler proof for commutative approximate inverse sequences.

Throughout the paper, we concentrate on compact metric spaces. Thus the systems that we deal with are so-called commutative approximate inverse sequences. This means that the bonding maps are commutative in the sense that $p_{i j} p_{j k}=p_{i k}$ for $i<j<k$. More general discussions on (noncommutative) approximate inverse systems and approximate maps can be found in [5], [7] and [8].

As an application, we relate approximate injectivity (resp., approximate surjectivity) to a monomorphism (resp., an epimorphism) in the approximate pro-category in the sense of [8]. We obtain characterizations of monomorphism and epimorphism in approximate pro-category. Monomorphisms and epimorphisms in pro-categories and pro*-categories were studied in $[2,1]$ (see [6, Ch. II, §2.1] for pro-groups).

Throughout the paper, map means continuous function unless otherwise stated. Let $\mathbb{N}$ denote the set of all positive integers.

\section{Approximate Sequences AND Approximate Resolutions}

Let $(X, d)$ be a metric space. Then, for each $\varepsilon>0$ and $A \subset X$, let $B(A, \varepsilon)=\{x \in X: d(x, a)<\varepsilon$ for some $a \in A\}$. 
For any $\varepsilon>0$ and $\delta>0$, a function $f: X \rightarrow Y$ between metric spaces is said to be $(\varepsilon, \delta)$-continuous if $d\left(x, x^{\prime}\right)<\delta$ implies $d\left(f(x), f\left(x^{\prime}\right)\right)<\varepsilon$ for all $x, x^{\prime} \in X$.

An approximate inverse sequence (approximate sequence, in short) $\left(X_{i}, \varepsilon_{i}\right.$, $\left.p_{i, i+1}\right)$ consists of compact metric spaces $X_{i}$, called coordinate spaces, positive real numbers $\varepsilon_{i}$, called meshes, and maps $p_{i, i+1}: X_{i+1} \rightarrow X_{i}$, called bonding maps, for $i \in \mathbb{N}$, and it must satisfy the following condition:

(A) for each $i \in \mathbb{N}$ and for each $\varepsilon>0$, there exists $i_{0}>i$ such that $p_{i i^{\prime}}$ is $\left(\varepsilon, \varepsilon_{i^{\prime}}\right)$-continuous for all $i^{\prime}>i_{0}$.

Here, we write $p_{i j}(i<j)$ for the composite $p_{i, i+1} p_{i+1, i+2} \cdots p_{j-1, j}$, and let $p_{i i}=1_{X_{i}}$.

An approximate map $\boldsymbol{p}=\left(p_{i}\right): X \rightarrow \mathfrak{X}$ of a compact metric space $X$ into an approximate sequence $\mathfrak{X}=\left(X_{i}, \varepsilon_{i}, p_{i, i+1}\right)$ consists of maps $p_{i}: X \rightarrow X_{i}$ for $i \in \mathbb{N}$, called projection maps, such that $p_{i}=p_{i j} p_{j}$ for $i<j$. It is an approximate resolution if it satisfies the following two conditions:

(R1) For each ANR $P, \varepsilon>0$ and map $f: X \rightarrow P$, there exist $i \in \mathbb{N}$ and a map $g: X_{i} \rightarrow P$ such that $d\left(g p_{i}, f\right)<\varepsilon$.

(R2) For each ANR $P$ and $\varepsilon>0$, there exists $\delta>0$ such that whenever $i \in \mathbb{N}$ and $g, g^{\prime}: X_{i} \rightarrow P$ are maps such that $d\left(g p_{i}, g^{\prime} p_{i}\right)<\delta$, then $d\left(g p_{i i^{\prime}}, g^{\prime} p_{i i^{\prime}}\right)<\varepsilon$ for some $i^{\prime}>i$.

For any approximate map $\boldsymbol{p}=\left(p_{i}\right): X \rightarrow \mathfrak{X}=\left(X_{i}, \varepsilon_{i}, p_{i, i+1}\right)$, consider the following conditions:

(B1) For each $\varepsilon>0$, there exists $i \in \mathbb{N}$ such that

$$
d\left(p_{i}(x), p_{i}\left(x^{\prime}\right)\right)<\varepsilon_{i} \Longrightarrow d\left(x, x^{\prime}\right)<\varepsilon, \text { for all } x, x^{\prime} \in X .
$$

(B2) For each $i \in \mathbb{N}$, there exits $i^{\prime}>i$ such that

$$
p_{i i^{\prime}}\left(X_{i^{\prime}}\right) \subset B\left(p_{i}(X), \varepsilon_{i}\right) .
$$

$(\mathrm{B} 1)^{*}$ For each $\varepsilon>0$, there exist $i \in \mathbb{N}$ and $\delta>0$ such that

$$
d\left(p_{i}(x), p_{i}\left(x^{\prime}\right)\right)<\delta \Longrightarrow d\left(x, x^{\prime}\right)<\varepsilon, \text { for all } x, x^{\prime} \in X .
$$

$(\mathrm{B} 2)^{*}$ For each $i \in \mathbb{N}$ and for each $\varepsilon>0$, there exits $i^{\prime}>i$ such that

$$
p_{i i^{\prime}}\left(X_{i^{\prime}}\right) \subset B\left(p_{i}(X), \varepsilon\right) .
$$

The following is a useful characterization of approximate resolution.

Theorem 2.1. For any approximate map $\boldsymbol{p}=\left(p_{i}\right): X \rightarrow \mathfrak{X}=$ $\left(X_{i}, \varepsilon_{i}, p_{i, i+1}\right)$, the following conditions are equivalent:

1) $\boldsymbol{p}$ is an approximate resolution of $X$.

2) $\boldsymbol{p}$ satisfies conditions (B1) and (B2).

3) $\boldsymbol{p}$ satisfies conditions $(\mathrm{B} 1)^{*}$ and $(\mathrm{B} 2)^{*}$. 
Proof. An approximate map $\boldsymbol{p}$ is an approximate resolution of $X$ if and only if the induced system map $\boldsymbol{p}=\left(p_{i}\right): X \rightarrow \boldsymbol{X}=\left(X_{i}, p_{i, i+1}\right)$ is a resolution in the sense of $[6$, p. 74$]$, which is characterized by conditions (B1)* and $(\mathrm{B} 2)^{*}$ (see $[6$, Theorems 3, 4, 5, Ch. I, §6.2]). Note here that our (B1)* and $(\mathrm{B} 2)^{*}$ are (B2) and (B1) in [6], respectively, and that the coverings in (B1) and (B2) of [6] can be replaced by positive real numbers since the spaces are compact metric spaces. Thus, we have 1) $\Leftrightarrow 3)$. Moreover, since (B1) $\Leftrightarrow$ $(\mathrm{B} 1)^{*}$ and $(\mathrm{B} 2) \Leftrightarrow(\mathrm{B} 2)^{*}$ hold, we have 2$\left.) \Leftrightarrow 3\right)$.

Recall that a system map $\boldsymbol{p}=\left(p_{i}\right): X \rightarrow \boldsymbol{X}=\left(X_{i}, p_{i, i+1}\right)$ (which means that $p_{i}$ 's satisfy $p_{i}=p_{i, i+1} p_{i+1}$ for each $\left.i \in \mathbb{N}\right)$ is a limit of $\boldsymbol{X}$ if it satisfies the following universal property:

$(\mathrm{UL})^{*}$ For any sysmte map $\boldsymbol{q}=\left(q_{i}\right): Y \rightarrow \boldsymbol{X}$ of a space, there exists a unique map $g: Y \rightarrow X$ such that $p_{i} g=q_{i}$ for each $i \in \mathbb{N}$.

In a similar way, an approximate map $\boldsymbol{p}=\left(p_{i}\right): X \rightarrow \mathfrak{X}=\left(X_{i}, \varepsilon_{i}, p_{i, i+1}\right)$ is defined to be a limit of $\mathfrak{X}$ if it satisfies the following universal property:

(UL) For any approximate map $\boldsymbol{q}=\left(q_{i}\right): Y \rightarrow \boldsymbol{X}$ of a space, there exists a unique map $g: Y \rightarrow X$ such that $p_{i} g=q_{i}$ for $i \in \mathbb{N}$.

If $\boldsymbol{p}: X \rightarrow \mathfrak{X}$ is a limit of $\mathfrak{X}$, then $X$ is determined up to homeomorphism. An approximate map $\boldsymbol{p}=\left(p_{i}\right): X \rightarrow \mathfrak{X}=\left(X_{i}, \varepsilon_{i}, p_{i, i+1}\right)$ is a limit of $\mathfrak{X}$ if and only if the induced system map $\boldsymbol{p}=\left(p_{i}\right): X \rightarrow \boldsymbol{X}=\left(X_{i}, p_{i, i+1}\right)$ is a limit.

The following theorem shows the existence of approximate resolution.

THEOREM 2.2. Every compact metric space $X$ admits an approximate resolution $\boldsymbol{p}=\left(p_{i}\right): X \rightarrow \mathfrak{X}=\left(X_{i}, \varepsilon_{i}, p_{i, i+1}\right)$ such that $X_{i}$ are compact polyhedra.

Proof. Every compact metric space $X$ admits an inverse sequence $\boldsymbol{X}=$ $\left(X_{i}, p_{i, i+1}\right)$ of compact polyhedra with limit $\boldsymbol{p}: X \rightarrow \boldsymbol{X}$ (see [6, Corollary 4, p. 62], for example). This $\boldsymbol{p}$ satisfies conditions (R1) and (R2) (see [6, Theorem 8, p. 63], for example), and there exist $\varepsilon_{i}>0(i \in \mathbb{N})$ such that $\mathfrak{X}=\left(X_{i}, \varepsilon_{i}, p_{i, i+1}\right)$ is an approximate sequence (see [9, Proposition 3.8]). Thus $\boldsymbol{p}=\left(p_{i}\right): X \rightarrow \mathfrak{X}$ defines an approximate resolution.

Throughout the paper, all the coordinate spaces $X_{i}$ of the approximate sequence $\mathfrak{X}$ are assumed to be compact polyhedra when we speak of an approximate resolution $\boldsymbol{p}: X \rightarrow \mathfrak{X}$.

An approximate map $\boldsymbol{f}=\left(f_{j}, \varphi\right): \mathfrak{X} \rightarrow \mathfrak{Y}$ between approximate sequences $\mathfrak{X}=\left(X_{i}, \varepsilon_{i}, p_{i, i+1}\right)$ and $\mathfrak{Y}=\left(Y_{j}, \delta_{j}, q_{j, j+1}\right)$ consists of an increasing function $\varphi: \mathbb{N} \rightarrow \mathbb{N}$ and maps $f_{j}: X_{\varphi(j)} \rightarrow Y_{j}$ for $j \in \mathbb{N}$, and it must satisfy the following condition:

(M) For any $j, j^{\prime} \in \mathbb{N}$ with $j<j^{\prime}$, there exists $i>\varphi\left(j^{\prime}\right)$ such that

$$
d\left(q_{j j^{\prime}} f_{j \prime} p_{\varphi\left(j^{\prime}\right) i}, f_{j} p_{\varphi(j) i^{\prime}}\right)<\delta_{j} .
$$


An approximate map $\boldsymbol{f}=\left(f_{j}, \varphi\right): \mathfrak{X} \rightarrow \mathfrak{Y}$ is said to be an approximate level map if $\varphi$ is the identity function on $\mathbb{N}$. if

A map $f: X \rightarrow Y$ is a limit of an approximate map $\boldsymbol{f}=\left(f_{j}, \varphi\right): \mathfrak{X} \rightarrow \mathfrak{Y}$

(L) For each $\varepsilon>0$ and for each $j \in \mathbb{N}$, there exists $j_{0}>j$ such that

$$
d\left(q_{j j^{\prime}} f_{j^{\prime}} p_{\varphi\left(j^{\prime}\right)}, q_{j} f\right)<\varepsilon, \text { for all } j^{\prime}>j_{0} .
$$

An approximate resolution of a map $f: X \rightarrow Y$ is a triple $(\boldsymbol{p}, \boldsymbol{q}, \boldsymbol{f})$ consisting of approximate resolutions $\boldsymbol{p}=\left(p_{i}\right): X \rightarrow \mathfrak{X}$ and $\boldsymbol{q}=\left(q_{j}\right): Y \rightarrow \mathfrak{Y}$ of $X$ and $Y$, respectively, and an approximate map $\boldsymbol{f}$ satisfying condition (L). The following theorem shows the existence of approximate resolution of a map for any choice of approximate resolutions (see [9, Theorem 4.3]).

Theorem 2.3. For any approximate resolutions $\boldsymbol{p}: X \rightarrow \mathfrak{X}$ and $\boldsymbol{q}: Y \rightarrow$ $\mathfrak{Y}$ of compact metric spaces $X$ and $Y$, respectively, every map $f: X \rightarrow Y$ admits an approximate map $\boldsymbol{f}: \mathfrak{X} \rightarrow \mathfrak{Y}$ such that $(\boldsymbol{p}, \boldsymbol{q}, \boldsymbol{f})$ is an approximate resolution of $f$.

\section{Approximate inJeCtivity of APPROXimate MAP}

In this section, we define the notion of approximate injectivity for approximate maps and show that this notion characterizes injective maps between compact metric spaces.

An approximate map $\boldsymbol{f}=\left(f_{j}, \varphi\right): \mathfrak{X} \rightarrow \mathfrak{Y}$ between approximate sequences $\mathfrak{X}=\left(X_{i}, \varepsilon_{i}, p_{i, i+1}\right)$ and $\mathfrak{Y}=\left(Y_{j}, \delta_{j}, q_{j, j+1}\right)$ is said to be approximately injective if it satisfies the following condition (see Diagram (3.1)):

(API) $(\forall i \in \mathbb{N})(\exists j \in \mathbb{N})\left(\exists j_{0}>j\right)\left(\forall j^{\prime}>j_{0}\right)\left(\exists i^{\prime}>\varphi\left(j^{\prime}\right), i\right)\left(\forall x, x^{\prime} \in X_{i^{\prime}}\right)$ :

$$
d\left(q_{j j^{\prime}} f_{j^{\prime}} p_{\varphi\left(j^{\prime}\right) i^{\prime}}(x), q_{j j^{\prime}} f_{j^{\prime}} p_{\varphi\left(j^{\prime}\right) i^{\prime}}\left(x^{\prime}\right)\right)<\delta_{j} \Longrightarrow d\left(p_{i i^{\prime}}(x), p_{i i^{\prime}}\left(x^{\prime}\right)\right)<\varepsilon_{i} .
$$

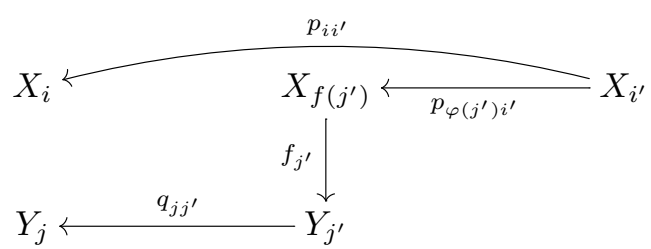

The main theorem states as follows.

TheOREM 3.1. Let $f: X \rightarrow Y$ be a map between compact metric spaces, and let $\boldsymbol{f}=\left(f_{j}, \varphi\right): \mathfrak{X} \rightarrow \mathfrak{Y}$ be an approximate map between approximate sequences $\mathfrak{X}=\left(X_{i}, \varepsilon_{i}, p_{i, i+1}\right)$ and $\mathfrak{Y}=\left(Y_{j}, \delta_{j}, q_{j, j+1}\right)$ such that $(\boldsymbol{p}, \boldsymbol{q}, \boldsymbol{f})$ is an approximate resolution of $f$, where $\boldsymbol{p}=\left(p_{i}\right): X \rightarrow \mathfrak{X}$ and $\boldsymbol{q}=\left(q_{j}\right): Y \rightarrow \mathfrak{Y}$ are approximate resolutions of $X$ and $Y$, respectively. Then $f$ is injective if and only if $\boldsymbol{f}$ is approximately injective. 
We break the proof into the following two lemmas.

Lemma 3.2. If a map $f: X \rightarrow Y$ is injective, then the approximate map $f$ is approximately injective.

Proof. Let $i \in \mathbb{N}$. It follows from the uniform continuity of $p_{i}$ on $X$, which is compact, that there exists $\xi_{1}>0$ such that

$$
d\left(x, x^{\prime}\right)<\xi_{1} \Longrightarrow d\left(p_{i}(x), p_{i}\left(x^{\prime}\right)\right)<\varepsilon_{i} / 3 \text {, for all } x, x^{\prime} \in X .
$$

The fact that the inverse of $f$ is uniformly continuous on the image of $f$ implies that there exists $\xi_{2}>0$ such that

$$
d\left(f(x), f\left(x^{\prime}\right)\right)<\xi_{2} \Longrightarrow d\left(x, x^{\prime}\right)<\xi_{1}, \text { for all } x, x^{\prime} \in X .
$$

Condition (B1) for $\boldsymbol{q}$ implies that there exists $j \in \mathbb{N}$ such that

$$
d\left(q_{j}(y), q_{j}\left(y^{\prime}\right)\right)<\delta_{j} \Longrightarrow d\left(y, y^{\prime}\right)<\xi_{2}, \text { for all } y, y^{\prime} \in Y .
$$

Condition (A) implies that there exists $j^{\prime \prime}>j$ such that

$$
d\left(y, y^{\prime}\right)<\delta_{j^{\prime \prime}} \Longrightarrow d\left(q_{j j^{\prime}}(y), q_{j j^{\prime}}\left(y^{\prime}\right)\right)<\delta_{j} / 9 \text {, for } y, y^{\prime} \in Y_{j^{\prime}} .
$$

Condition (L) for $j$ and $\delta_{j} / 3$ implies that there exists $j_{0}>j^{\prime \prime}$ such that

$$
d\left(q_{j j^{\prime}} f_{j^{\prime}} p_{\varphi\left(j^{\prime}\right)}, q_{j} f\right)<\delta_{j} / 3 \text { for } j^{\prime}>j_{0} .
$$

Fix $j^{\prime}>j_{0}$. Then we have

Claim 1. For any $x, x^{\prime} \in X$,

$$
d\left(q_{j j^{\prime}} f_{j^{\prime}} p_{\varphi\left(j^{\prime}\right)}(x), q_{j j^{\prime}} f_{j^{\prime}} p_{\varphi\left(j^{\prime}\right)}\left(x^{\prime}\right)\right)<\delta_{j} / 3
$$

implies

$$
d\left(p_{i}(x), p_{i}\left(x^{\prime}\right)\right)<\varepsilon_{i} / 3 .
$$

Indeed, (3.6) and (3.7) imply

$$
d\left(q_{j} f(x), q_{j} f\left(x^{\prime}\right)\right)<\delta_{j} .
$$

This together with (3.4), (3.3) and (3.2) implies (3.8).

Now take $i^{\prime} \in \mathbb{N}$ such that $i^{\prime}>i, \varphi\left(j^{\prime}\right)$. By uniform continuity, there exists $\eta_{1}>0$ such that for all $x, x^{\prime} \in X_{i^{\prime}}$,

(3.9) $d\left(x, x^{\prime}\right)<\eta_{1} \Longrightarrow d\left(q_{j j^{\prime}} f_{j^{\prime}} p_{\varphi\left(j^{\prime}\right) i^{\prime}}(x), q_{j j^{\prime}} f_{j^{\prime}} p_{\varphi\left(j^{\prime}\right) i^{\prime}}\left(x^{\prime}\right)\right)<\delta_{j} / 9$, and

$$
d\left(x, x^{\prime}\right)<\eta_{1} \Longrightarrow d\left(p_{i i^{\prime}}(x), p_{i i^{\prime}}\left(x^{\prime}\right)\right)<\varepsilon_{i} / 3 .
$$

Condition (B2) implies that there exists $i^{\prime \prime}>i^{\prime}$ such that

$$
p_{i^{\prime} i^{\prime \prime}}\left(X_{i^{\prime \prime}}\right) \subset B\left(p_{i^{\prime}}(X), \eta_{1}\right) .
$$

Claim 2. For any $x, x^{\prime} \in X_{i^{\prime \prime}}$,

$$
d\left(q_{j j^{\prime}} f_{j^{\prime}} p_{\varphi\left(j^{\prime}\right) i^{\prime \prime}}(x), q_{j j^{\prime}} f_{j^{\prime}} p_{\varphi\left(j^{\prime}\right) i^{\prime \prime}}\left(x^{\prime}\right)\right)<\delta_{j} / 9
$$

implies

$$
d\left(p_{i i^{\prime \prime}}(x), p_{i i^{\prime \prime}}\left(x^{\prime}\right)\right)<\varepsilon_{i} .
$$


Let $x, x^{\prime} \in X_{i^{\prime \prime}}$ satisfy (3.12). (3.11) implies that there exist $z, z^{\prime} \in X$ such that

$$
d\left(p_{i^{\prime} i^{\prime \prime}}(x), p_{i^{\prime}}(z)\right)<\eta_{1} \text {, and } d\left(p_{i^{\prime} i^{\prime \prime}}\left(x^{\prime}\right), p_{i^{\prime}}\left(z^{\prime}\right)\right)<\eta_{1},
$$

respectively. This together with (3.9) implies

$$
\begin{aligned}
d\left(q_{j j^{\prime}} f_{j^{\prime}} p_{\varphi\left(j^{\prime}\right) i^{\prime \prime}}(x), q_{j j^{\prime}} f_{j^{\prime}} p_{\varphi\left(j^{\prime}\right)}(z)\right) & <\delta_{j} / 9, \text { and } \\
d\left(q_{j j^{\prime}} f_{j^{\prime}}\right. & \left.p_{\varphi\left(j^{\prime}\right) i^{\prime \prime}}\left(x^{\prime}\right), q_{j j^{\prime}} f_{j^{\prime}} p_{\varphi\left(j^{\prime}\right)}\left(z^{\prime}\right)\right)<\delta_{j} / 9 .
\end{aligned}
$$

(3.12) and (3.15) imply

$$
d\left(q_{j j^{\prime}} f_{j^{\prime}} p_{\varphi\left(j^{\prime}\right)}(z), q_{j j^{\prime}} f_{j^{\prime}} p_{\varphi\left(j^{\prime}\right)}\left(z^{\prime}\right)\right)<\delta_{j} / 3 .
$$

This together with Claim 1 implies

$$
d\left(p_{i}(z), p_{i}\left(z^{\prime}\right)\right)<\varepsilon_{i} / 3 .
$$

(3.14) and (3.10) imply

$$
d\left(p_{i i^{\prime \prime}}(x), p_{i}(z)\right)<\varepsilon_{i} / 3 \text {, and } d\left(p_{i i^{\prime \prime}}\left(x^{\prime}\right), p_{i}\left(z^{\prime}\right)\right)<\varepsilon_{i} / 3 .
$$

(3.16) and (3.17) then imply (3.13), as required (see Diagram (3.18)).

Now (3.5) and Claim 2 imply that for any $x, x^{\prime} \in X_{i^{\prime \prime}}$,

$$
d\left(q_{j^{\prime \prime} j^{\prime}} f_{j^{\prime}} p_{\varphi\left(j^{\prime}\right) i^{\prime \prime}}(x), q_{j^{\prime \prime} j^{\prime}} f_{j^{\prime}} p_{\varphi\left(j^{\prime}\right) i^{\prime \prime}}\left(x^{\prime}\right)\right)<\delta_{j^{\prime \prime}} \Longrightarrow d\left(p_{i i^{\prime \prime}}(x), p_{i i^{\prime \prime}}\left(x^{\prime}\right)\right)<\varepsilon_{i} .
$$

Then, for $j:=j^{\prime \prime}, j_{0}$ and $i^{\prime}:=i^{\prime \prime}$, the condition (API) is fulfilled. This proves that $\boldsymbol{f}$ is approximately injective.

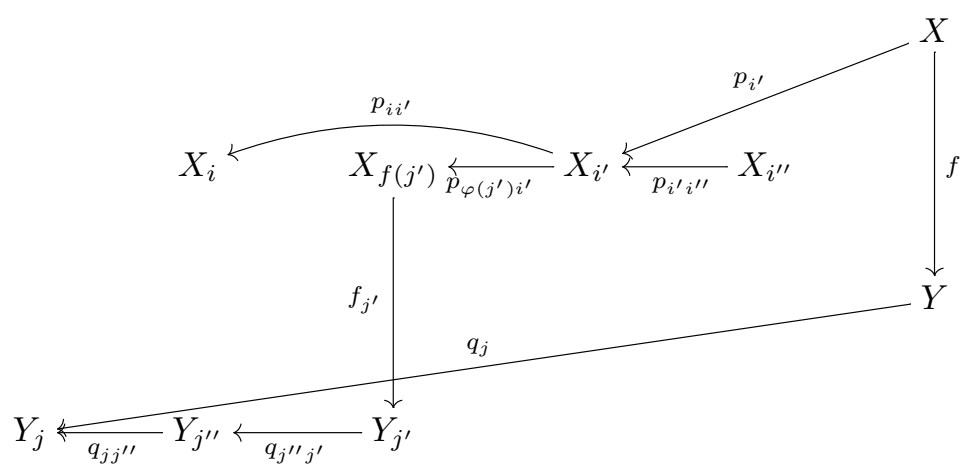

Lemma 3.3. If the approximate map $\boldsymbol{f}$ is approximately injective, then the map $f$ is injective.

Proof. Suppose that $\boldsymbol{f}$ satisfies condition (API). Let $\varepsilon>0$. Condition (B1) for $\boldsymbol{p}$ implies that there exists $i \in \mathbb{N}$ such that

$$
d\left(p_{i}(x), p_{i}\left(x^{\prime}\right)\right)<\varepsilon_{i} \Longrightarrow d\left(x, x^{\prime}\right)<\varepsilon .
$$


Condition (API) implies that there exist $j \in \mathbb{N}$ and $j_{0} \in \mathbb{N}$ with $j_{0}>j$ such that each $j^{\prime}>j_{0}$ admits $i^{\prime}>\varphi\left(j^{\prime}\right), i$ with

$$
\begin{aligned}
d\left(q_{j j^{\prime}} f_{j^{\prime}} p_{\varphi\left(j^{\prime}\right) i^{\prime}}(x), q_{j j^{\prime}} f_{j^{\prime}} p_{\varphi\left(j^{\prime}\right) i^{\prime}}\left(x^{\prime}\right)\right)<\delta_{j} \\
\Longrightarrow d\left(p_{i i^{\prime}}(x), p_{i i^{\prime}}\left(x^{\prime}\right)\right)<\varepsilon_{i}, \text { for all } x, x^{\prime} \in X_{i^{\prime}} .
\end{aligned}
$$

Condition (L) implies that there exists $j^{\prime}>j_{0}$ such that

$$
d\left(q_{j j^{\prime}} f_{j^{\prime}} p_{\varphi\left(j^{\prime}\right)}, q_{j} f\right)<\delta_{j} / 3 .
$$

The uniform continuity of $q_{j}$ implies that there exist $\eta>0$ such that

$$
d\left(y, y^{\prime}\right)<\eta \Longrightarrow d\left(q_{j}(y), q_{j}\left(y^{\prime}\right)\right)<\delta_{j} / 3 \text { for all } y, y^{\prime} \in Y .
$$

We have

Claim. For any $x, x^{\prime} \in X, d\left(f(x), f\left(x^{\prime}\right)\right)<\eta \Longrightarrow d\left(x, x^{\prime}\right)<\varepsilon$.

Suppose that $x, x^{\prime} \in X$ and $d\left(f(x), f\left(x^{\prime}\right)\right)<\eta$. Then this together with (3.22) implies

$$
d\left(q_{j} f(x), q_{j} f\left(x^{\prime}\right)<\delta_{j} / 3\right.
$$

This and (3.21) imply

$$
d\left(q_{j j^{\prime}} f_{j^{\prime}} p_{\varphi\left(j^{\prime}\right)}(x), q_{j j^{\prime}} f_{j^{\prime}} p_{\varphi\left(j^{\prime}\right)}\left(x^{\prime}\right)\right)<\delta_{j}
$$

By (3.20), this then implies

$$
d\left(p_{i}(x), p_{i}\left(x^{\prime}\right)\right)<\varepsilon_{i} .
$$

This together with (3.19) implies

$$
d\left(x, x^{\prime}\right)<\varepsilon
$$

as required.

The claim implies that if $f(x)=f\left(x^{\prime}\right)$ then $d\left(x, x^{\prime}\right)<\varepsilon$ for any $\varepsilon>0$, showing that $x=x^{\prime}$. This proves that $f$ is injective.

\section{Approximate surJectivity of approximate map}

In this section, we define the notion of approximate surjectivity for approximate maps and show that this notion characterizes surjective maps between compact metric spaces.

An approximate map $\boldsymbol{f}=\left(f_{j}, \varphi\right): \mathfrak{X} \rightarrow \mathfrak{Y}$ between approximate sequences $\mathfrak{X}=\left(X_{i}, \varepsilon_{i}, p_{i, i+1}\right)$ and $\mathfrak{Y}=\left(Y_{j}, \delta_{j}, q_{j, j+1}\right)$ is said to be approximately surjective if it satisfies the following condition (see Diagram (4.1)):

(APS) $(\forall j \in \mathbb{N})\left(\exists j_{0}>j\right)\left(\forall j^{\prime}>j_{0}\right)\left(\exists j^{\prime \prime}>j^{\prime}\right)\left(\exists i_{0}>\varphi\left(j^{\prime}\right)\right)\left(\forall i>i_{0}\right)(\forall y \in$ $\left.Y_{j^{\prime \prime}}\right)\left(\exists x \in X_{i}\right)$ :

$$
d\left(q_{j j^{\prime \prime}}(y), q_{j j^{\prime}} f_{j^{\prime}} p_{\varphi\left(j^{\prime}\right) i}(x)\right)<\delta_{j} .
$$




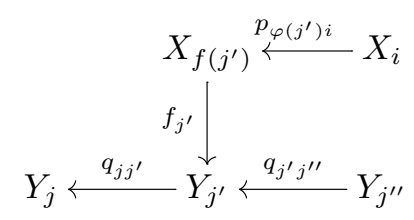

ThEOREM 4.1. Let $f: X \rightarrow Y$ be a map between compact metric spaces, and let $\boldsymbol{f}=\left(f_{j}, \varphi\right): \mathfrak{X} \rightarrow \mathfrak{Y}$ be an approximate map between approximate sequences $\mathfrak{X}=\left(X_{i}, \varepsilon_{i}, p_{i, i+1}\right)$ and $\mathfrak{Y}=\left(Y_{j}, \delta_{j}, q_{j, j+1}\right)$ such that $(\boldsymbol{p}, \boldsymbol{q}, \boldsymbol{f})$ is an approximate resolution of $f$, where $\boldsymbol{p}=\left(p_{i}\right): X \rightarrow \mathfrak{X}$ and $\boldsymbol{q}=\left(q_{j}\right): Y \rightarrow \mathfrak{Y}$ are approximate resolutions of $X$ and $Y$, respectively. Then $f$ is surjective if and only if $\boldsymbol{f}$ is approximately surjective.

We break the proof into the following two lemmas.

Lemma 4.2. If a map $f: X \rightarrow Y$ is surjective, then the approximate map $f$ is approximately surjective.

Proof. Let $\varepsilon>0$ and $j \in \mathbb{N}$. Condition (A) for $\boldsymbol{q}$ implies that there exists $j_{0}>j$ such that

$$
d\left(y, y^{\prime}\right)<\delta_{j^{\prime}} \Longrightarrow d\left(q_{j j^{\prime}}(y), q_{j j^{\prime}}\left(y^{\prime}\right)\right)<\delta_{j} / 3 \text {, for } j^{\prime}>j_{0} \text { and } y, y^{\prime} \in Y_{j^{\prime}} .
$$

Fix $j^{\prime}>j_{0}$. Then condition (B2) for $\boldsymbol{q}$ and condition (L) imply that there exists $j^{\prime \prime}>j^{\prime}$ such that

$$
\begin{gathered}
q_{j^{\prime} j^{\prime \prime}}\left(Y_{j^{\prime \prime}}\right) \subset B\left(q_{j^{\prime}}(Y), \delta_{j^{\prime}}\right), \text { and } \\
d\left(q_{j^{\prime}} f, q_{j^{\prime} j^{\prime \prime}} f_{j^{\prime \prime}} p_{\varphi\left(j^{\prime \prime}\right)}\right)<\delta_{j^{\prime}} .
\end{gathered}
$$

Condition (M) implies that there exists $i_{0}>\varphi\left(j^{\prime \prime}\right)$ such that

$$
d\left(f_{j^{\prime}} p_{\varphi\left(j^{\prime}\right) i}, q_{j^{\prime} j^{\prime \prime}} f_{j^{\prime \prime}} p_{\varphi\left(j^{\prime \prime}\right) i}\right)<\delta_{j^{\prime}}, \text { for } i>i_{0} .
$$

Now let $y \in Y_{j^{\prime \prime}}$. (4.3) implies that there exists $y^{\prime} \in Y$ such that

$$
d\left(q_{j^{\prime} j^{\prime \prime}}(y), q_{j^{\prime}}\left(y^{\prime}\right)\right)<\delta_{j^{\prime}} .
$$

Since $f$ is surjective, there exists $x \in X$ such that $f(x)=y^{\prime}$. Claim that

$$
d\left(q_{j j^{\prime \prime}}(y), q_{j j^{\prime}} f_{j^{\prime}} p_{\varphi\left(j^{\prime}\right) i}\left(p_{i}(x)\right)\right)<\delta_{j}, \text { for } i>i_{0} .
$$

Indeed, (4.4) implies that

$$
d\left(q_{j^{\prime}} f(x), q_{j^{\prime} j^{\prime \prime}} f_{j^{\prime \prime}} p_{\varphi\left(j^{\prime \prime}\right)}(x)\right)<\delta_{j^{\prime}} .
$$

(4.5) implies that

$$
d\left(f_{j^{\prime}} p_{\varphi\left(j^{\prime}\right) i}\left(p_{i}(x)\right), q_{j^{\prime} j^{\prime \prime}} f_{j^{\prime \prime}} p_{\varphi\left(j^{\prime \prime}\right) i}\left(p_{i}(x)\right)\right)<\delta_{j^{\prime}}, \text { for } i>i_{0} .
$$


(4.6), (4.8), (4.9) together with (4.2) imply (4.7) as required (see Diagram $(4.10))$. This proves that $\boldsymbol{f}$ is approximately surjective.

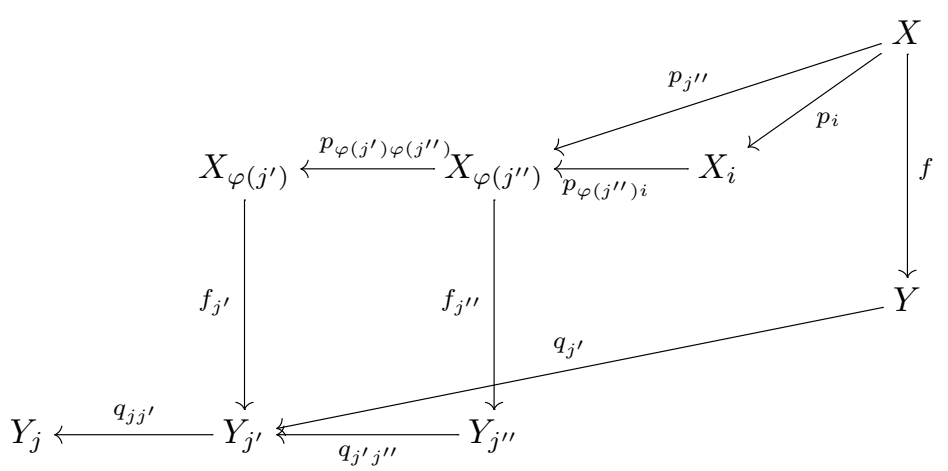

LEMMA 4.3. If the approximate map $\boldsymbol{f}$ is approximately surjective, then the map $f$ is surjective.

Proof. Let $y \in Y$. For each $j \in \mathbb{N}$, put $y_{j}=q_{j}(y)$. We wish to find $x \in X$ such that $y=f(x)$.

Conditions (APS), (M), and (A) imply that there exist subsequences $\left\{j_{k}\right\}$, $\left\{i_{k}\right\}$ of $\mathbb{N}$, and points $z_{i_{k}} \in X_{i_{k}}(k \in \mathbb{N})$ such that $j_{k}<j_{k+1}, \varphi\left(j_{k}\right)<i_{k}<$ $\varphi\left(j_{k+1}\right)$, and the following three conditions hold (see Diagram (4.14)):

$$
\begin{gathered}
d\left(y_{j_{k}}, q_{j_{k} j_{k+1}} f_{j_{k+1}} p_{\varphi\left(j_{k+1}\right) i_{k+1}}\left(z_{i_{k+1}}\right)\right)<\delta_{j_{k}}, \\
d\left(f_{j_{k}} p_{\varphi\left(j_{k}\right) i_{n}}, q_{j_{k} j_{n}} f_{j_{n}} p_{\varphi\left(j_{n}\right) i_{n}}\right)<\delta_{j_{k}}, \text { for } n>k, \text { and }
\end{gathered}
$$
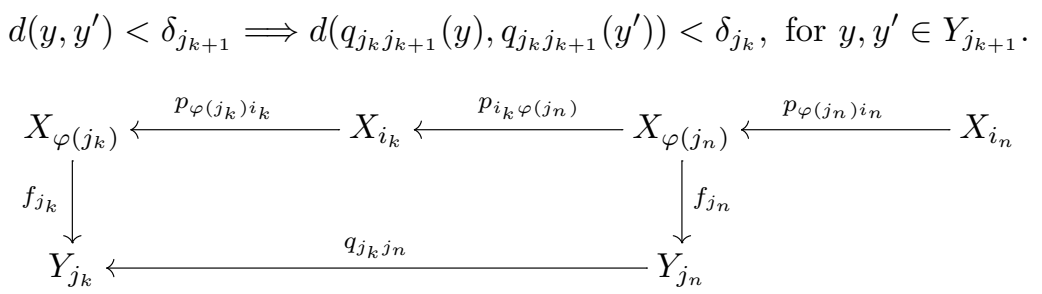

Replace $\mathfrak{X}$ by the subsequence $\mathfrak{X}^{\prime}=\left(X_{i_{k}}, p_{i_{k} i_{k+1}}\right)$, $\mathfrak{Y}$ by the subsequence $\mathfrak{Y}^{\prime}=\left(Y_{j_{k}}, q_{j_{k} j_{k+1}}\right)$, and $\boldsymbol{f}$ by the approximate level map $\boldsymbol{f}^{\prime}=\left(f_{k}^{\prime}\right)$ where $f_{k}^{\prime}=f_{j_{k}} p_{\varphi\left(j_{k}\right) i_{k}}$, and assume that $\boldsymbol{f}=\left(f_{j}\right): \mathfrak{X} \rightarrow \mathfrak{Y}$ is an approximate level map satisfying the following two conditions (see Diagram (4.18)):

$$
\begin{gathered}
d\left(y_{k}, q_{k, k+1} f_{k+1}\left(z_{k+1}\right)\right)<\delta_{k}, \\
d\left(f_{k} p_{k n}, q_{k n} f_{n}\right)<\delta_{k}, \text { for } n>k, \text { and }
\end{gathered}
$$

$$
d\left(y, y^{\prime}\right)<\delta_{k+1} \Longrightarrow d\left(q_{k, k+1}(y), q_{k, k+1}\left(y^{\prime}\right)\right)<\delta_{k}, \text { for } y, y^{\prime} \in Y_{k+1} .
$$




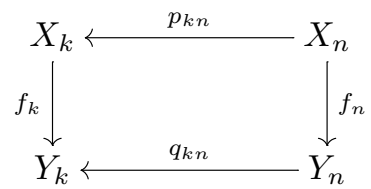

Since each $X_{k}$ is compact, one can find a decreasing sequence of infinite subsets of $\mathbb{N}, I_{1} \supset I_{2} \supset \cdots$, such that $I_{k+1}$ is cofinal in $I_{k}$, and

$$
\left\{p_{k i}\left(z_{i}\right)\right\}_{i \in I_{k}} \text { converges to some point } x_{k} \in X_{k} .
$$

CLAIM. For each $k \in \mathbb{N}, x_{k}=\lim _{n \rightarrow \infty} p_{k n}\left(x_{n}\right)$.

To see this, let $\varepsilon>0$. Then (4.19) and condition (A) imply that there exists $N \in \mathbb{N}$ such that for each $n \geq N$,

$$
d\left(x_{k}, p_{k n}\left(z_{n}\right)\right)<\varepsilon / 2 \text {, and }
$$

$$
d\left(x, x^{\prime}\right)<\varepsilon_{n} \Longrightarrow d\left(p_{k n}(x), p_{k n}\left(x^{\prime}\right)\right)<\varepsilon / 2 \text {, for all } x, x^{\prime} \in X_{n} .
$$

For each $n \geq N$, there exists $m \geq n$ such that

$$
d\left(x_{n}, p_{n m}\left(z_{m}\right)\right)<\varepsilon_{n} .
$$

This together with (4.21) implies

$$
d\left(p_{k n}\left(x_{n}\right), p_{k m}\left(z_{m}\right)\right)<\varepsilon / 2 .
$$

(4.20) and (4.22) then imply

$$
d\left(x_{k}, p_{k n}\left(x_{n}\right)\right)<\varepsilon,
$$

proving the claim.

The claim means that the sequence $\left(x_{k}\right)$ forms a thread and determines a point $x \in X$. We show $f(x)=y$. To see this, let $j \in \mathbb{N}$ and $\varepsilon>0$. Conditions (A) and (L) imply that there exists $k \in \mathbb{N}$ such that

$$
\begin{gathered}
d\left(y, y^{\prime}\right)<\delta_{k} \Longrightarrow \\
d\left(q_{j k}(y), q_{j k}\left(y^{\prime}\right)\right)<\varepsilon / 4, \text { for } y, y^{\prime} \in Y_{k}, \text { and } \\
d\left(q_{j k} f_{k} p_{k}, q_{j} f\right)<\varepsilon / 4 .
\end{gathered}
$$

By uniform continuity, there exists $\delta>0$ such that

$$
d\left(z, z^{\prime}\right)<\delta \Longrightarrow d\left(q_{j k} f_{k}(z), q_{j k} f_{k}\left(z^{\prime}\right)\right)<\varepsilon / 4, \text { for } z, z^{\prime} \in X_{k} .
$$

There exists $n>k$ (see (4.19)) such that

$$
d\left(x_{k}, p_{k n}\left(z_{n}\right)\right)<\delta .
$$

This together with (4.25) implies

$$
d\left(q_{j k} f_{k}\left(x_{k}\right), q_{j k} f_{k} p_{k n}\left(z_{n}\right)\right)<\varepsilon / 4 .
$$

(4.16) and (4.23) imply

$$
d\left(q_{j k} f_{k} p_{k n}, q_{j n} f_{n}\right)<\varepsilon / 4 .
$$


(4.15), (4.17) and (4.23) imply

$$
d\left(y_{j}, q_{j n} f_{n}\left(z_{n}\right)\right)<\varepsilon / 4 .
$$

By (4.24), (4.26), (4.27), (4.28) (see Diagram (4.29)),

$$
d\left(y_{j}, q_{j} f(x)\right)<\varepsilon .
$$

This shows that $f(x)=y$.

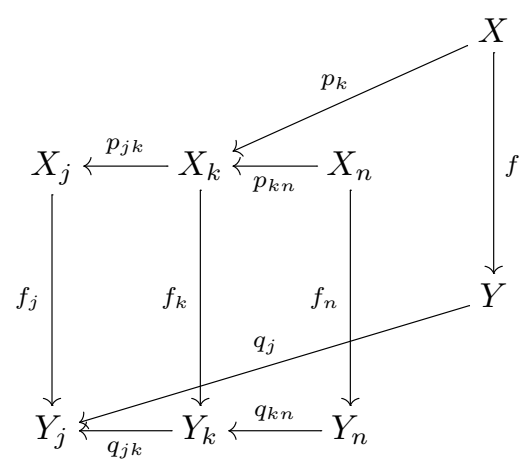

5. Conditions equivalent to (API) And (APS)

In this section, we discuss some variations of Theorems 3.1 and 4.1.

Given any approximate sequence $\mathfrak{X}=\left(X_{i}, \varepsilon_{i}, p_{i, i+1}\right)$, forgetting the numbers $\varepsilon_{i}$, we obtain an inverse sequence $\boldsymbol{X}=\left(X_{i}, p_{i, i+1}\right)$. Conversely, given any inverse sequence $\boldsymbol{X}=\left(X_{i}, p_{i, i+1}\right)$, there exist $\varepsilon_{i}>0$ for $i \in \mathbb{N}$ such that $\mathfrak{X}=\left(X_{i}, \varepsilon_{i}, p_{i, i+1}\right)$ is an approximate sequence (see [9, Proposition 3.8]). Thus we are interested in conditions (API) and (APS) without using meshes for approximate sequences.

For any approximate sequence $\mathfrak{X}=\left(X_{i}, \varepsilon_{i}, p_{i, i+1}\right)$, consider the following two conditions:

$(\mathrm{API})^{*}(\forall \varepsilon>0)(\forall i \in \mathbb{N})(\exists \delta>0)(\exists j \in \mathbb{N})\left(\exists j_{0}>j\right)\left(\forall j^{\prime}>j_{0}\right)\left(\exists i^{\prime}>\right.$ $\left.\varphi\left(j^{\prime}\right), i\right)\left(\forall x, x^{\prime} \in X_{i^{\prime}}\right)$ :

$d\left(q_{j j^{\prime}} f_{j^{\prime}} p_{\varphi\left(j^{\prime}\right) i^{\prime}}(x), q_{j j^{\prime}} f_{j^{\prime}} p_{\varphi\left(j^{\prime}\right) i^{\prime}}\left(x^{\prime}\right)\right)<\delta \Longrightarrow d\left(p_{i i^{\prime}}(x), p_{i i^{\prime}}\left(x^{\prime}\right)\right)<\varepsilon$.

and

$(\mathrm{APS})^{*}(\forall \varepsilon>0)(\forall j \in \mathbb{N})\left(\exists j_{0}>j\right)\left(\forall j^{\prime}>j_{0}\right)\left(\exists j^{\prime \prime}>j^{\prime}\right)\left(\exists i_{0}>\varphi\left(j^{\prime}\right)\right)(\forall i>$ $\left.i_{0}\right)\left(\forall y \in Y_{j^{\prime \prime}}\right)\left(\exists x \in X_{i}\right)$ :

$$
d\left(q_{j j^{\prime \prime}}(y), q_{j j^{\prime}} f_{j^{\prime}} p_{\varphi\left(j^{\prime}\right) i}(x)\right)<\varepsilon .
$$

The following proposition shows the equivalence between conditions (API) and $(\mathrm{API})^{*}$. 
Proposition 5.1. Let $\boldsymbol{f}=\left(f_{j}, f\right): \mathfrak{X} \rightarrow \mathfrak{Y}$ be an approximate map between approximate sequences $\mathfrak{X}=\left(X_{i}, \varepsilon_{i}, p_{i, i+1}\right)$ and $\mathfrak{Y}=\left(Y_{j}, \delta_{j}, q_{j, j+1}\right)$. Then $\boldsymbol{f}$ satisfies condition (API) if and only if $\boldsymbol{f}$ satisfies condition (API)*

Proof. Suppose that $\mathfrak{X}$ satisfies condition (API). For each $\varepsilon>0$ and $i \in \mathbb{N}$, there exists $i^{\prime}>i$ such that

$$
d\left(x, x^{\prime}\right)<\delta_{i^{\prime}} \Longrightarrow d\left(p_{i i^{\prime}}(x), p_{i i^{\prime}}\left(x^{\prime}\right)\right)<\varepsilon, \text { for } x, x^{\prime} \in X_{i^{\prime}} .
$$

Apply condition (API) for this $i^{\prime}$ to obtain $j \in \mathbb{N}$ and $j_{0}>j$ as in (API). Then condition (API)* holds with $\delta=\delta_{j}$.

Conversely, suppose that $\mathfrak{X}$ satisfies condition (API)* Let $i \in \mathbb{N}$, and for this $i$ and $\varepsilon=\varepsilon_{i}$, take $\delta>0, j \in \mathbb{N}$, and $j_{0}>j$ as in (API)*. Condition (A) implies that there exists $j^{\prime}>j_{0}$ such that

$$
d\left(y, y^{\prime}\right)<\delta_{j^{\prime}} \Longrightarrow d\left(q_{j j^{\prime}}(y), q_{j j^{\prime}}\left(y^{\prime}\right)\right)<\delta / 3 \text {, for } y, y^{\prime} \in Y_{j^{\prime}} .
$$

Let $j^{\prime \prime}>j^{\prime}$. Then condition (M) implies that there exists $i^{\prime}>\varphi\left(j^{\prime \prime}\right)$ such that

$$
d\left(f_{j^{\prime}} p_{\varphi\left(j^{\prime}\right) i^{\prime}}, q_{j^{\prime} j^{\prime \prime}} f_{j^{\prime \prime}} p_{\varphi\left(j^{\prime \prime}\right) i^{\prime}}\right)<\delta_{j^{\prime}} .
$$

Claim that for any $x, x^{\prime} \in X_{i^{\prime}}$,

$$
d\left(q_{j^{\prime} j^{\prime \prime}} f_{j^{\prime \prime}} p_{\varphi\left(j^{\prime \prime}\right) i^{\prime}}(x), q_{j^{\prime} j^{\prime \prime}} f_{j^{\prime \prime}} p_{\varphi\left(j^{\prime \prime}\right) i^{\prime}}\left(x^{\prime}\right)\right)<\delta_{j^{\prime}}
$$

implies

$$
d\left(p_{i i^{\prime}}(x), p_{i i^{\prime}}\left(x^{\prime}\right)\right)<\varepsilon_{i} .
$$

Indeed, (5.3) and (5.1) imply

$$
d\left(q_{j j^{\prime \prime}} f_{j^{\prime \prime}} p_{\varphi\left(j^{\prime \prime}\right) i^{\prime}}(x), q_{j j^{\prime \prime}} f_{j^{\prime \prime}} p_{\varphi\left(j^{\prime \prime}\right) i^{\prime}}\left(x^{\prime}\right)\right)<\delta / 3,
$$

and (5.2) and (5.1) imply

$$
d\left(q_{j j^{\prime}} f_{j^{\prime}} p_{\varphi\left(j^{\prime}\right) i^{\prime}}, q_{j j^{\prime \prime}} f_{j^{\prime \prime}} p_{\varphi\left(j^{\prime \prime}\right) i^{\prime}}\right)<\delta / 3 .
$$

Those two inequalities imply

$$
d\left(q_{j j^{\prime}} f_{j^{\prime}} p_{\varphi\left(j^{\prime}\right) i^{\prime}}(x), q_{j j^{\prime}} f_{j^{\prime}} p_{\varphi\left(j^{\prime}\right) i^{\prime}}\left(x^{\prime}\right)\right)<\delta .
$$

This together with condition (API)* then implies (5.4). This verifies condition (API).

The following proposition shows the equivalence between conditions (APS) and (APS)*.

Proposition 5.2. Let $\boldsymbol{f}=\left(f_{j}, f\right): \mathfrak{X} \rightarrow \mathfrak{Y}$ be an approximate map between approximate sequences $\mathfrak{X}=\left(X_{i}, \varepsilon_{i}, p_{i, i+1}\right)$ and $\mathfrak{Y}=\left(Y_{j}, \delta_{j}, q_{j, j+1}\right)$. Then $\boldsymbol{f}$ satisfies condition (APS) if and only if $\boldsymbol{f}$ satisfies condition (APS)* ${ }^{*}$. 
Proof. Suppose that an approximate map $f: \mathfrak{X} \rightarrow \mathfrak{Y}$ satisfies condition (APS). For each $\varepsilon>0$ and $j \in \mathbb{N}$, condition (A) implies that there exists $j^{\prime}>j$ such that

$$
d\left(y, y^{\prime}\right)<\delta_{j^{\prime}} \Longrightarrow d\left(q_{j j^{\prime}}(y), q_{j j^{\prime}}\left(y^{\prime}\right)\right)<\varepsilon, \text { for all } y, y^{\prime} \in Y_{j^{\prime}} .
$$

Apply (APS) with this $j^{\prime}$ to get (APS)*. The converse is obvious.

\section{MONOMORPHISMS AND EPIMORPHISMS IN APPROXIMATE PRO-CATEGORIES}

In this section, we obtain characterizations of monomorphism and epimorphism in the approximate pro-category. More precisely, we show that condition (API) (resp., (APS)) gives a characterization of a monomorphism (resp., an epimorphism) in the approximate pro-category. For this purpose, we use the categorical equivalence of the approximate pro-category and the topological category.

First, we recall the definition of approximate pro-category. Our version of approximate pro-category (restricted for the class of compact metric spaces) is a little simpler than the definitions in $[9, \S 2]$ and [8].

Let $\mathcal{C}$ be any full subcategory of the category $\mathrm{CM}$ of compact metric spaces. For two approximate maps $\boldsymbol{f}=\left(f_{j}, \varphi\right), \boldsymbol{f}^{\prime}=\left(f_{j}^{\prime}, \varphi^{\prime}\right): \mathfrak{X} \rightarrow \mathfrak{Y}$ between approximate sequences $\mathfrak{X}=\left(X_{i}, \varepsilon_{i}, p_{i, i+1}\right)$ and $\mathfrak{Y}=\left(Y_{j}, \delta_{j}, q_{j, j+1}\right)$ in $\mathcal{C}$, we define a relation $\sim$ by setting $\boldsymbol{f} \sim \boldsymbol{f}^{\prime}$ if and only if each $j \in \mathbb{N}$ admits $i>\varphi(j), \varphi^{\prime}(j)$ such that

$$
d\left(f_{j} p_{\varphi(j) i}, f_{j}^{\prime} p_{\varphi^{\prime}(j) i}\right)<\delta_{j} .
$$

We then define a relation $\equiv$ by setting $\boldsymbol{f} \equiv \boldsymbol{f}^{\prime}$ if and only if there exist finitely many approximate maps $\boldsymbol{f}_{i}: \mathfrak{X} \rightarrow \mathfrak{Y}, i=1,2, \ldots, n$, such that $\boldsymbol{f}=\boldsymbol{f}_{1}$, $\boldsymbol{f}_{i} \sim \boldsymbol{f}_{i+1}$ for $i=1,2, \ldots, n-1$, and $\boldsymbol{f}^{\prime}=\boldsymbol{f}_{n}$. Then the relation $\equiv$ is an equivalence relation, and the equivalence class of $\boldsymbol{f}$ is denoted by $[\boldsymbol{f}]$.

The objects of APRO-C are approximate sequences in $\mathcal{C}$. The set APRO- $\mathcal{C}(\mathfrak{X}, \mathfrak{Y})$ of morphisms $\mathfrak{X} \rightarrow \mathfrak{Y}$ is the set of the equivalence classes of uniform approximate maps $\mathfrak{X} \rightarrow \mathfrak{Y}$ with respect to the equivalence relation $\equiv$. Here, an approximate map $\boldsymbol{f}=\left(f_{j}, \varphi\right): \mathfrak{X} \rightarrow \mathfrak{Y}$ is uniform if for each $j \in \mathbb{N}$,

$$
d\left(x, x^{\prime}\right)<\varepsilon_{\varphi(j)} \Longrightarrow d\left(f_{j}(x), f_{j}\left(x^{\prime}\right)\right)<\delta_{j}, \text { for } x, x^{\prime} \in X_{\varphi(j)} .
$$

Note that each approximate map $\boldsymbol{f}: \mathfrak{X} \rightarrow \mathfrak{Y}$ admits a uniform approximate $\operatorname{map} \boldsymbol{f}^{\prime}: \mathfrak{X} \rightarrow \mathfrak{Y}$ such that $\boldsymbol{f} \sim \boldsymbol{f}^{\prime}$.

For any uniform approximate maps $\boldsymbol{f}=\left(f_{j}, \varphi\right): \mathfrak{X} \rightarrow \mathfrak{Y}$ and $\boldsymbol{g}=\left(g_{k}, \psi\right)$ : $\mathfrak{Y} \rightarrow \mathfrak{Z}$, define the composition $[\boldsymbol{g}] \circ[\boldsymbol{f}]$ as the equivalence class of the uniform approximate map $\boldsymbol{h}=\left(h_{j}, \rho\right): \mathfrak{X} \rightarrow \mathfrak{Z}$ defined as in the following Proposition (see Appendix for its proof). 
Proposition 6.1. Let $\mathfrak{X}=\left(X_{i}, \varepsilon_{i}, p_{i, i+1}\right), \mathfrak{Y}=\left(Y_{j}, \delta_{j}, q_{j, j+1}\right)$, and $\mathfrak{X}=$ $\left(Z_{k}, \zeta_{k}, r_{k, k+1}\right)$ be approximate sequences in $\mathcal{C}$.

1. Let $s: \mathbb{N} \rightarrow \mathbb{N}$ be an increasing function which satisfies $s(k)>k$ for each $k \in \mathbb{N}$ and the following three conditions:

- for each $k^{\prime} \geq s(k)$,

$d\left(z, z^{\prime}\right)<4 \zeta_{k^{\prime}} \Longrightarrow d\left(r_{k k^{\prime}}(z), r_{k k^{\prime}}\left(z^{\prime}\right)\right)<\zeta_{k}$, for $z, z^{\prime} \in Z_{k^{\prime}}$,

- for each $k \in \mathbb{N}$,

$$
\begin{aligned}
& d\left(z, z^{\prime}\right)<\zeta_{s(k+1)} \\
& \quad \Longrightarrow d\left(r_{s(k) s(k+1)}(z), r_{s(k) s(k+1)}\left(z^{\prime}\right)\right)<\zeta_{s(k)}, \text { for } z, z^{\prime} \in Z_{s(k+1)}, \\
& \quad \text { and }
\end{aligned}
$$

For each $k \in \mathbb{N}$, define a map $h_{k}: X_{\varphi(\psi(s(k)))} \rightarrow Z_{k}$ by $h_{k}=$ $r_{k s(k)} g_{s(k)} f_{\psi(s(k))}$. Then $\boldsymbol{h}=\left(h_{k}\right): \mathfrak{X} \rightarrow \mathfrak{Z}$ defines a uniform approximate map.

2. Let $\boldsymbol{f}=\left(f_{j}, \varphi\right), \boldsymbol{f}^{\prime}=\left(f_{j}^{\prime}, \varphi^{\prime}\right): \mathfrak{X} \rightarrow \mathfrak{Y}$ and $\boldsymbol{g}=\left(g_{k}, \psi\right), \boldsymbol{g}^{\prime}=\left(g_{k}^{\prime}, \psi^{\prime}\right)$ : $\mathfrak{Y} \rightarrow \mathfrak{Z}$ be uniform approximate maps, and let $\boldsymbol{h}=\left(h_{k}, \rho\right), \boldsymbol{h}^{\prime}=$ $\left(h_{k}^{\prime}, \rho^{\prime}\right): \mathfrak{X} \rightarrow \mathfrak{Z}$ be the uniform approximate maps that are defined by $\boldsymbol{f}$ and $\boldsymbol{g}, \boldsymbol{f}^{\prime}$ and $\boldsymbol{g}^{\prime}$, respectively, as in 1). Then if $\boldsymbol{f} \sim \boldsymbol{f}^{\prime}$ and $\boldsymbol{g} \sim \boldsymbol{g}^{\prime}$, then $\boldsymbol{h} \sim \boldsymbol{h}^{\prime}$.

Let the identity $\operatorname{id}_{\mathfrak{X}} \in \mathrm{APRO}-\mathcal{C}(\mathfrak{X}, \mathfrak{X})$ be the equivalence class which is represented by the approximate map $1_{\mathfrak{X}}=\left(1_{X_{j}}, 1_{\mathbb{N}}\right)$. Thus defined objects and morphisms together with the composition and the identity form a category, which is called the category of approximate systems in $\mathcal{C}$ and denoted by APRO-C.

Let $\mathrm{CPol}$ be the full subcategory of $\mathrm{CM}$ whose objects are compact polyhedra. Let lim be the limit functor APRO-CPol $\rightarrow$ CM. More precisely, each approximate sequence $\mathfrak{X}=\left(X_{i}, \varepsilon_{i}, p_{i, i+1}\right)$ in CPol admits a nonempty compact metric space $X$ together with an approximate resolution $\boldsymbol{p}=\left(p_{i}\right): X \rightarrow \mathfrak{X}$. Let $\lim \mathfrak{X}$ be the space $X$. Each uniform approximate map $\boldsymbol{f}=\left(f_{j}, \varphi\right): \mathfrak{X} \rightarrow \mathfrak{Y}$ admits a limit map $f=\lim \boldsymbol{f}: X \rightarrow Y$ between the limits $X=\lim \mathfrak{X}$ and $Y=\lim \mathfrak{Y}$. For any two uniform approximate maps $\boldsymbol{f}, \boldsymbol{f}^{\prime}: \mathfrak{X} \rightarrow \mathfrak{Y}$, if $\boldsymbol{f} \sim \boldsymbol{f}^{\prime}$, then $\lim \boldsymbol{f}=\lim \boldsymbol{f}^{\prime}$ (see [8, Theorem 7.7]). So we can define $\lim [\boldsymbol{f}]$ as the limit $f: X \rightarrow Y$ of the equivalence class $[\boldsymbol{f}]$. Then $\lim$ is functorial and preserves the identities, and thus lim is a functor.

THEOREM 6.2. The functor $\mathrm{lim}: \mathrm{APRO}-\mathrm{CPol} \rightarrow \mathrm{CM}$ is an equivalence of categories.

Proof. It suffices to verify that the functor lim is faithful, full, and dense. Indeed, for any two uniform approximate maps $f, f^{\prime}: \mathfrak{X} \rightarrow \mathfrak{Y}$, if $\lim \boldsymbol{f}=\lim \boldsymbol{f}^{\prime}$, then $\boldsymbol{f} \sim \boldsymbol{f}^{\prime}$ (lim is faithful) (see [8, Theorem 7.7]). Each map 
$f: X \rightarrow Y$ with uniform approximate resolutions $\boldsymbol{p}: X \rightarrow \mathfrak{X}$ and $\boldsymbol{q}: Y \rightarrow \mathfrak{Y}$ admits a uniform approximate map $\boldsymbol{f}: \mathfrak{X} \rightarrow \mathfrak{Y}$ whose limit is $f$ (lim is full) (see Theorem 2.2). For each compact metric space $X$, there exists a uniform approximate resolution $\boldsymbol{p}: X \rightarrow \mathfrak{X}$, so that $X=\lim \mathfrak{X}$ (lim is dense) (see Theorem 2.3).

The following shows that the property of being approximately injective (resp., surjective) is defined in the approximate pro-category.

Proposition 6.3. Let $\boldsymbol{f}, \boldsymbol{g}: \boldsymbol{X} \rightarrow \boldsymbol{Y}$ be uniform approximate maps such that $\boldsymbol{f} \sim \boldsymbol{g}$. If $\boldsymbol{f}$ is approximately injective (resp., approximately surjective), then so is $\boldsymbol{g}$.

Proof. The statement follows from the fact that $\lim \boldsymbol{f}=\lim \boldsymbol{g}$, and Theorem 3.1 (resp., Theorem 4.1).

The following gives a characterization of monomorphism (resp., epimorphism) in the approximate pro-category.

THEOREM 6.4. For any approximate map $\boldsymbol{f}=\left(f_{j}, \varphi\right): \mathfrak{X} \rightarrow \mathfrak{Y}$, the morphism $[\boldsymbol{f}]$ is a monomorphism (resp., an epimorphism) in APRO-CPol if and only if $\boldsymbol{f}$ is approximately injective (resp., approximately surjective).

Proof. Let $f: X \rightarrow Y$ be a limit map of $\boldsymbol{f}$ with approximate resolutions $\boldsymbol{p}: X \rightarrow \mathfrak{X}$ and $\boldsymbol{q}: Y \rightarrow \mathfrak{Y}$. Consider the following conditions:

1. $f$ is a monomorphism (resp., an epimorphism) in CM,

2. $f$ is injective (resp., surjective),

3. $\boldsymbol{f}$ is approximately injective (resp., approximately surjective).

4. $[\boldsymbol{f}]$ is a monomorphism (resp., an epimorphism) in APRO-CPol, 1) and 2) are equivalent, 2) and 3) are equivalent (see Theorem 3.1 (resp., Theorem 4.1)), and 3) and 4) are equivalent (see Theorem 6.2). This shows the assertion.

\section{Appendix. Proof of Proposition 6.1}

Proof. To see part 1), let $k<k^{\prime}$. By (AM) for $\boldsymbol{g}$, there exists $j>$ $\psi\left(s\left(k^{\prime}\right)\right)$ such that

$$
d\left(g_{s(k)} q_{\psi(s(k)) j}, r_{s(k) s\left(k^{\prime}\right)} g_{s\left(k^{\prime}\right)} q_{\psi\left(s\left(k^{\prime}\right)\right) j}\right)<\zeta_{s(k)},
$$

and there exists $i>\varphi(j)$ such that

$$
\begin{array}{r}
d\left(f_{\psi(s(k))} p_{\varphi(\psi(s(k))) i}, q_{\psi(s(k)) j} f_{j} p_{\varphi(j) i}\right)<\delta_{\psi(s(k))}, \text { and } \\
d\left(f_{\psi\left(s\left(k^{\prime}\right)\right)} p_{\varphi\left(\psi\left(s\left(k^{\prime}\right)\right)\right) i}, q_{\psi\left(s\left(k^{\prime}\right)\right) j} f_{j} p_{\varphi(j) i}\right)<\delta_{\psi\left(s\left(k^{\prime}\right)\right)} .
\end{array}
$$

Then, by (A.2) and (6.1),

$$
d\left(\psi_{s(k)} f_{\psi(s(k))} p_{\varphi(\psi(s(k))) i}, g_{s(k)} q_{\psi(s(k)) j} f_{j} p_{\varphi(j) i}\right)<\zeta_{s(k)} .
$$


By (A.1),

(A.5) $\quad d\left(g_{s(k)} q_{\psi(s(k)) j} f_{j} p_{\varphi(j) i}, r_{s(k) s\left(k^{\prime}\right)} g_{s\left(k^{\prime}\right)} q_{\psi\left(s\left(k^{\prime}\right)\right) j} f_{j} p_{\varphi(j) i}\right)<\zeta_{s(k)}$.

By (A.3), (6.1), and (6.3),

(A.6)

$d\left(r_{s(k) s\left(k^{\prime}\right)} g_{s\left(k^{\prime}\right)} q_{\psi\left(s\left(k^{\prime}\right)\right) j} f_{j} p_{\varphi(j) i}, r_{s(k) s\left(k^{\prime}\right)} g_{s\left(k^{\prime}\right)} f_{\psi\left(s\left(k^{\prime}\right)\right)} p_{\varphi\left(\psi\left(s\left(k^{\prime}\right)\right)\right) i}\right)<\zeta_{s(k)}$.

(A.4), (A.5), (A.6) together with (6.2) imply

$d\left(r_{k s(k)} g_{s(k)} f_{\psi(s(k))} p_{\varphi(\psi(s(k))) i}, r_{k s\left(k^{\prime}\right)} g_{s\left(k^{\prime}\right)} f_{\psi\left(s\left(k^{\prime}\right)\right)} p_{\varphi\left(\psi\left(s\left(k^{\prime}\right)\right)\right) i}\right)<\zeta_{k}$,

showing that $\boldsymbol{h}$ satisfies condition (M) (see Diagram (A.7)). That $\boldsymbol{h}$ is uniform follows from (6.4) and the assumption that both $\boldsymbol{f}$ and $\boldsymbol{g}$ are uniform.

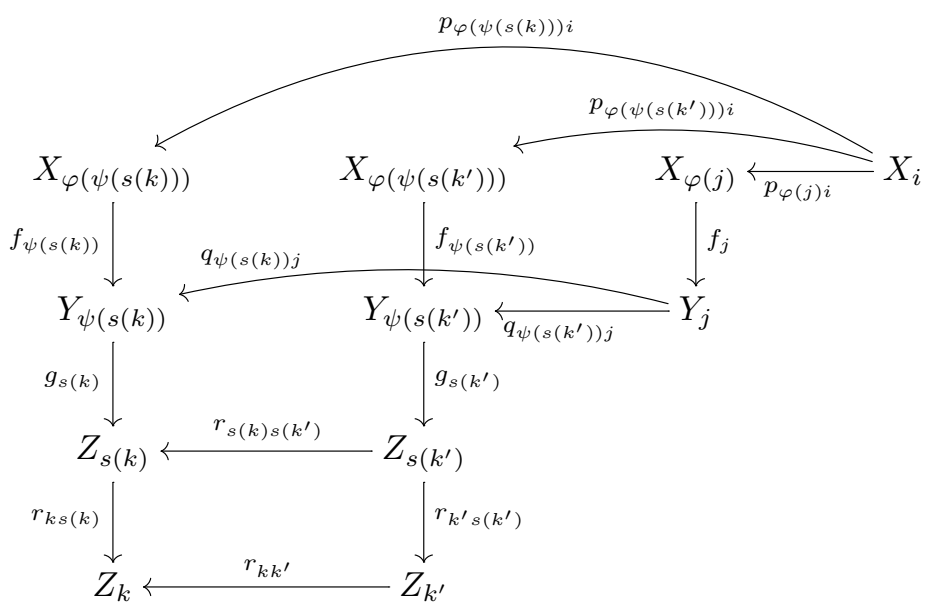

To see part 2), let $k \in \mathbb{N}$. Then $\boldsymbol{g} \sim \boldsymbol{g}^{\prime}$ and condition (A) imply that there exists $j>\psi(s(k)), \psi^{\prime}(s(k))$ such that

$$
d\left(g_{s(k)} q_{\psi(s(k)) j}, g_{s(k)}^{\prime} q_{\psi^{\prime}(s(k)) j}\right)<\zeta_{s(k)},
$$

$$
\begin{aligned}
d\left(y, y^{\prime}\right)< & \delta_{j} \Longrightarrow d\left(q_{\psi(s(k)) j}(y), q_{\psi(s(k)) j}\left(y^{\prime}\right)\right)<\delta_{\psi(s(k))}, \text { and } \\
d\left(y, y^{\prime}\right)< & \delta_{j} \Longrightarrow \\
& \quad d\left(q_{\psi^{\prime}(s(k)) j}(y), q_{\psi^{\prime}(s(k)) j}\left(y^{\prime}\right)\right)<\delta_{\psi^{\prime}(s(k))} \text { for } y, y^{\prime} \in Y_{j} .
\end{aligned}
$$

Moreover, $\boldsymbol{f} \sim \boldsymbol{f}^{\prime}$ and condition $(\mathrm{AM})$ for $\boldsymbol{f}$ and $\boldsymbol{f}^{\prime}$ imply that there exists $i>\varphi(j), \varphi^{\prime}(j)$ such that

$$
d\left(f_{j} p_{\varphi(j) i}, f_{j}^{\prime} p_{\varphi^{\prime}(j) i}\right)<\delta_{j},
$$

$$
d\left(f_{\psi(s(k))} p_{\varphi(\psi(s(k))) i}, q_{\psi(s(k)) j} f_{j} p_{\varphi(j) i}\right)<\delta_{\psi(s(k))},
$$

$$
d\left(f_{\psi^{\prime}(s(k))}^{\prime} p_{\varphi^{\prime}\left(\psi^{\prime}(s(k))\right) i}, q_{\psi^{\prime}(s(k)) j} f_{j}^{\prime} p_{\varphi^{\prime}(j) i}\right)<\delta_{\psi^{\prime}(s(k))} .
$$


(A.12) implies

$$
d\left(g_{s(k)} f_{\psi(s(k))} p_{\varphi(\psi(s(k))) i}, g_{s(k)} q_{\psi(s(k)) j} f_{j} p_{\varphi(j) i}\right)<\zeta_{s(k)} .
$$

(A.11) and (A.9) imply

$$
d\left(g_{s(k)} q_{\psi(s(k)) j} f_{j} p_{\varphi(j) i}, g_{s(k)} q_{\psi(s(k)) j} f_{j}^{\prime} p_{\varphi^{\prime}(j) i}\right)<\zeta_{s(k)} .
$$

(A.8) implies

$$
d\left(g_{s(k)} q_{\psi(s(k)) j} f_{j}^{\prime} p_{\varphi^{\prime}(j) i}, g_{s(k)}^{\prime} q_{\psi^{\prime}(s(k)) j} f_{j}^{\prime} p_{\varphi^{\prime}(j) i}\right)<\zeta_{s(k)} .
$$

(A.13) implies

$$
d\left(g_{s(k)}^{\prime} q_{\psi^{\prime}(s(k)) j} f_{j}^{\prime} p_{\varphi^{\prime}(j) i}, g_{s(k)}^{\prime} f_{\psi^{\prime}(s(k))}^{\prime} p_{\varphi^{\prime}\left(\psi^{\prime}(s(k))\right) i}\right)<\zeta_{s(k)} .
$$

(A.14), (A.15), (A.16), (A.17) imply

$$
d\left(g_{s(k)} f_{\psi(s(k))} p_{\varphi(\psi(s(k))) i}, g_{s(k)}^{\prime} f_{\psi^{\prime}(s(k))}^{\prime} p_{\varphi^{\prime}\left(\psi^{\prime}(s(k))\right) i}\right)<4 \zeta_{s(k)}
$$

(see Diagram (A.18)). This together with (6.2) implies

$$
d\left(r_{k s(k)} g_{s(k)} f_{\psi(s(k))} p_{\varphi(\psi(s(k))) i}, r_{k s(k)} g_{s(k)}^{\prime} f_{\psi^{\prime}(s(k))}^{\prime} p_{\varphi^{\prime}\left(\psi^{\prime}(s(k))\right) i}\right)<\zeta_{k} .
$$

This shows $\boldsymbol{h} \sim \boldsymbol{h}^{\prime}$.

(A.18)

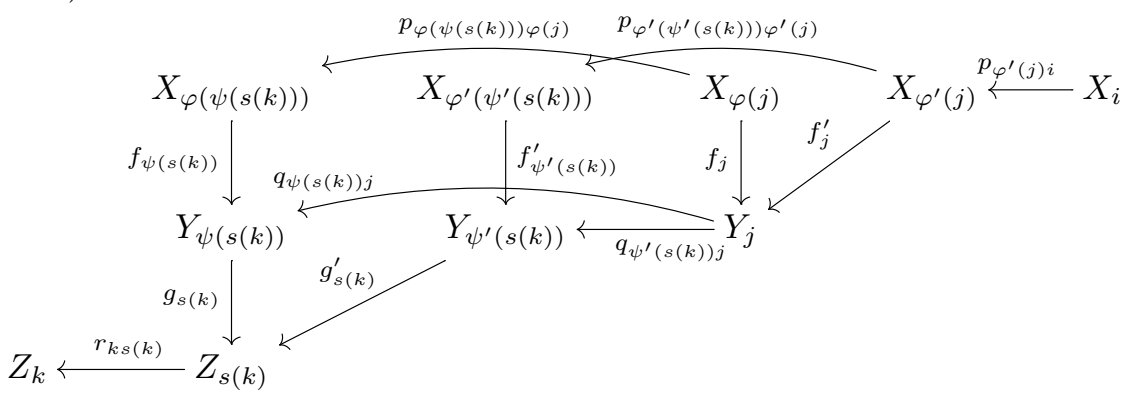

\section{REFERENCES}

[1] N. K. Bilan, Comparing monomorphisms and epimorphisms in pro and pro*categories, Topol. Appl. 155 (2008), 1840-1851.

[2] J. Dydak and F. R. Ruiz del Portal, Monomorphisms and epimorphisms in procategories, Topol. Appl. 154 (2007), 2204-2222.

[3] T. Miyata and T. Watanabe, Approximate resolutions of the fractal category, Glas. Mat. Ser. III 38 (2003), 377-393.

[4] S. Mardešić, Approximate polyhedra, resolutions of maps and shape fibrations, Fund. Math. 114 (1981), 53-78.

[5] S. Mardešić and L. Rubin, Approximate inverse systems of compacta and covering dimension, Pacific J. Math. 138 (1989), 129-144.

[6] S. Mardešić and J. Segal, Shape Theory, North-Holland, Amsterdam-New York, 1982.

[7] S. Mardešić and J. Segal, Mapping approximate inverse systems of compacta, Fund. Math. 134 (1990), 73-91. 
[8] S. Mardešić and T. Watanabe, Approximate resolutions of spaces and maps, Glas. Mat. Ser. III 24 (1989), 587-637.

[9] T. Watanabe, Approximative shape I, Tsukuba J. Math. 11 (1987), 17-59.

T. Miyata

Department of Mathematics and Informatics

Graduate School of Human Development and Environment

Kobe University

Kobe, 657-8501

Japan

E-mail: tmiyata@kobe-u.ac.jp

Received: 11.4.2016.

Revised: 24.10.2016. 